\title{
ENDOSCOPÍA TRAQUEAL EN EL DIAGNÓSTICO DE CASOS SUBCLÍNICOS DE HEMORRAGIA PULMONAR INDUCIDA POR EJERCICIO EN CABALLOS DE CARRERA
}

\author{
Tracheal Endoscopy on the Diagnosis of Pulmonary Hemorraghycal \\ Subclinical Cases Induced by Exercise in Racing Horses
}

Jean Hon Y. ${ }^{1}$, Sergio Cueva M. ${ }^{1,2}$, César Alzamora P. ${ }^{3}$ y Julio Arias R. ${ }^{4}$

\section{Resumen}

El objetivo del presente estudio fue determinar el porcentaje de presentación de Hemorragia Pulmonar Inducida por Ejercicio (HPIE) mediante examen endoscópico en caballos Pura Sangre de Carrera después de realizada una competencia. Se utilizó 172 caballos de 2 a 8 años de edad ( 75 hembras y 97 machos) del Hipódromo de Monterrico de Lima. La evaluación endoscópica traqueal fue realizada entre los 30 y 90 minutos de reposo posteriores a la carrera. Los animales con sangre en la tráquea fueron considerados positivos. El 59.9\% de animales fue positivo a HPIE, sin haber diferencias significativas en la presentación de HPIE entre sexos y tipos de pista de carrera.

Palabras clave: Hemorragia Pulmonar Inducida por Ejercicio, HPIE, caballos de carrera, endoscopía traqueal

\section{Abstract}

This study aimed to determine the percentage of Exercise-Induced Pulmonary Hemorrhage (EIPH) by endoscopic evaluation after racing in Thoroughbred horses. A total of 172 horses between 2 to 8 years old ( 75 females and 97 males) at Monterrico racetrack in Lima were used. The endoscopic tracheal evaluation was done between 30 and 90 minutes of rest after the race. The animals that had blood in the trachea were considered positive. The results showed that $59.9 \%$ of the horses were positive to EIPH, but without significant differences due to sex and type of race track.

Key words: Exercise-Induced Pulmonary Hemorrhage, EIPH, Thoroughbred horses, tracheal endoscopy

\footnotetext{
${ }^{1}$ Laboratorio de Fisiología Animal, ${ }^{3}$ Laboratorio de Zootecnia y Producción Agropecuaria, Facultad de Medicina Veterinaria, Universidad Nacional Mayor de San Marcos, Lima

${ }^{2}$ E-mail:scuevam@unmsm.edu.pe

${ }^{4}$ Hipódromo de Monterrico, Lima
} 


\section{INTRODUCCIÓN}

La hemorragia pulmonar inducida por ejercicio (HPIE) fue descrita por primera vez en 1688 (Morán y Araya, 2003). Afecta a más del 50\% de los caballos Pura Sangre de Carrera (Erickson, 2000), así como a otras razas como los Pony Club Horses, Standarbreds, Quarter Horses, Steeplechasers y Polo Ponies (Sellnow, 1997).

Hay diversas teorías que tratan de explicar la causa subyacente de la hemorragia de los capilares pulmonares. Se sabe que la presión de la arteria pulmonar aumenta dramáticamente durante el ejercicio a medida que el animal incrementa el esfuerzo (West et al., 1993; Erickson, 2000). En un estudio por microscopía electrónica se muestra una alteración de los capilares pulmonares, incluyendo interrupciones del endotelio capilar y de la capa epitelial alveolar, glóbulos rojos en la pared intersticial del alvéolo, fluidos proteináceos y glóbulos rojos en el espacio alveolar (West et al., 1993).

Se afirma que velocidades mayores a $14 \mathrm{~m} / \mathrm{s}$, desarrolladas por el equino durante el galope, son suficientes para que ocurra un aumento de la presión intrapulmonar capilar que puede romper estos vasos (Andresen, 1992; Colahan et al., 1998). Otra teoría indica que el golpeteo de los miembros delanteros durante la competencia causa un trauma en el tejido pulmonar (Geor, 2001).

Dentro de las causas de la presentación de HPIE, se puede considerar, además, a la infestación parasitaria, enfermedad pulmonar crónica, patógenos sanguíneos, obstrucción parcial de las vías aéreas superiores, defectos de la coagulación, asfixia y mecanismos de estrés en el parénquima pulmonar durante el ejercicio fuerte (West et al., 1993).

Esta enfermedad se diagnostica principalmente mediante la observación endoscópica de sangre dentro de la vía aé- rea superior y tráquea del caballo (Colahan et al., 1998). La endoscopía puede realizarse inmediatamente después del ejercicio vigoroso o competencia, aunque la experiencia clínica sugiere que el examen a los 30-90 minutos del ejercicio aumenta la probabilidad de observar sangre en las vías aéreas (Colahan et al., 1998; Geor, 2001).

\section{Materiales y Métodos}

El estudio se realizó en enero del 2004 en el Hipódromo de Monterrico, Lima. Se practicó la endoscopía a 172 caballos Pura Sangre de Carrera, de dos a ocho años de edad, de ambos sexos, luego de una carrera oficial. Los animales fueron seleccionados al azar y se esperó entre 30 a 90 minutos después de la carrera para iniciar la endoscopía.

Se utilizó un endoscopio flexible de fibra óptica marca Olympus GIF tipo XQ de 1 $\mathrm{m}$ de longitud y $0.8 \mathrm{~cm}$ de diámetro, con fuente de luz artificial halógena. Al animal, luego de su respectivo vareo y tiempo de espera, se le hacía ingresar al área de revisión y se le ubicaba dentro de un box o pesebrera. Previa sujeción por el vareador, mediante un filete, se le colocaba un acial en el belfo superior y se le introducía el endoscopio por uno de los ollares, de preferencia por el izquierdo, hasta llegar a la tráquea para determinar la presencia o ausencia de sangre (Fig. 1). Se registró, además, el sexo y tipo de pista (arena o césped).

El análisis estadístico se realizó mediante la prueba de chi cuadrado, con un nivel de significancia de 0.05 , para las variables sexo y tipo de pista.

\section{Resultados y Discusión}

El 59.9\% (103/172) de los equinos resultó positivo a HPIE en la evaluación endoscópica, sin encontrar diferencias estadísticas entre 

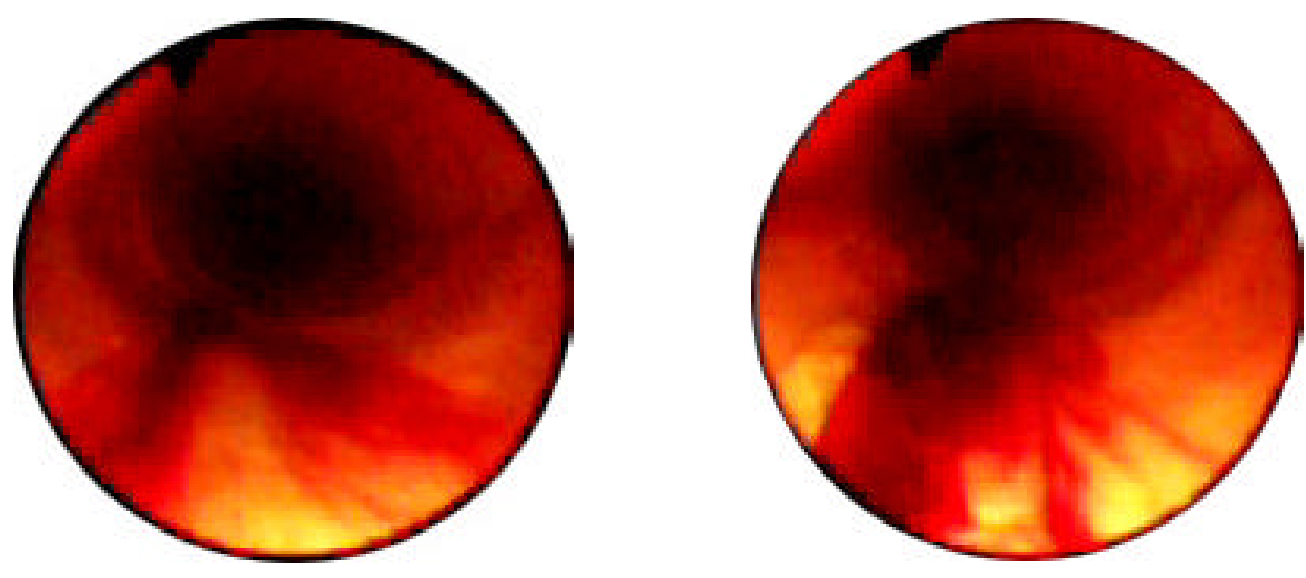

Figura 1. Hemorragia pulmonar inducida por ejercicio vista por endoscopía después de una carrera.

Cuadro 1. Frecuencia de animales positivos a hemorragia pulmonar inducida por el ejercicio en caballos de carrera, según sexo o tipo de pista de carrera

\begin{tabular}{clcccccc}
\hline & \multicolumn{3}{c}{ Positivos } & \multicolumn{3}{c}{ Negativos } \\
\hline & & $\mathrm{N}$ & $\%$ & I.C. & $\mathrm{n}$ & $\%$ & I.C. $^{1}$ \\
\hline \multirow{2}{*}{ Sexo } & Hembras & 47 & 62.7 & $50.7-73.6$ & 28 & 37.3 & $26.4-49.3$ \\
& Machos & 56 & 57.5 & $47.3-67.7$ & 41 & 42.3 & $32.2-52.7$ \\
& & & & & & & \\
\multirow{2}{*}{ Pista } & Arena & 66 & 60.6 & $50.7-69.8$ & 43 & 39.4 & $30.2-49.3$ \\
& Césped & 37 & 58.7 & $45.6-71.0$ & 26 & 41.3 & $29.0-54.4$ \\
\hline \multirow{2}{*}{ Total } & & 103 & 59.9 & $52.1-67.3$ & 69 & 40.1 & $32.7-47.9$ \\
\hline
\end{tabular}

${ }^{1}$ Intervalo de confianza del $95 \%$

sexos o tipo de pista de carrera (Cuadro 1). Este valor fue similar a las frecuencias de $50-70 \%$ reportadas en otros estudios (Rojas et al., 2000; Geor et al., 2001); sin embargo, es bastante alto respecto a reportes previos en el medio, donde Zamalloa (1979) y Jiménez (2000) mencionan frecuencias de 6.1 y $1.2 \%$ obtenidos a través de observaciones de epistaxis. Esto resalta la importancia del uso de la endoscopía en el diagnóstico de la HPIE.
Los reportes sobre el sexo como factor predisponente no son concluyentes. Los trabajos locales muestran tendencias a una mayor ocurrencia de HPIE en hembras (Zamalloa, 1979; Jiménez, 2000), en tanto que otros estudios muestran una mayor frecuencia, aunque no significativa, en machos castrados (Rojas et al., 2000; Morán et al., 2003), posiblemente debido a la disminución de la eritropoyesis y síntesis de hemoglobina por la disminución de los niveles de testosterona. 
Se considera que el impacto de los cascos en la pista de competencia crea ondas que repercuten en los pulmones, sobre todo en la zona dorso lumbar (Geor, 2001). De ser así, se esperaría que las carreras en pistas duras aumenten la generación de ondas causantes de la ruptura de los capilares pulmonares. En el Perú, básicamente se dispone de dos tipos de pistas: la de césped y la de arena, siendo la primera un terreno más duro en comparación con la segunda, ya que las pistas de arena son constantemente removidas para evitar su compactación. No obstante, los resultados encontrados indicaron una mayor ocurrencia de HPIE en pista de arena, aunque sin diferencias estadísticas entre ambas pistas.

\section{Conclusiones}

? Mediante la endoscopía traqueal se detectó que la Hemorragia Pulmonar Inducida por el Ejercicio (HPIE) se presentó en el 59.9\% de los caballos de carrera.

? El sexo y el tipo de pista no fueron factores predisponentes en la presentación de la HPIE.

\section{Litirkatura Citada}

1. Andresen H. 1992. Manual de las enfermedades de los equinos. Lima: La Moderna.

2. Colahan PT, Mayhew IG, Merritt AM, Moore JN. 1998. Medicina y cirugía equina. $4^{\text {a }}$ ed. Vol 1. Argentina: Intermédica.

3. Erickson H. 2000. A review of exercise-induced pulmonary hemorrhage and new concepts for prevention. En: AAEP Proceedings. USA. 46: 193-196.
4. Geor R. 2001. With every breath he takes. EIPH: Exercise-induced pulmonary hemorrhage. The Horse, USA 18(11): 87-92.

5. Jiménez E. 2000. Factores de riesgo en la presentación de hemorragia pulmonar inducida por esfuerzo (EIPH) en caballos de carrera. Tesis de Médico Veterinario. Lima: Facultad de Medicina Veterinaria, Univ. Nacional Mayor de San Marcos. 39 p.

6. Morán G, Araya O. 2003. Hemorragia pulmonar inducida por el ejercicio en el caballo: una revisión. Arch Med Vet, Valdivia 35(2): 127-138.

7. Morán G, Carrillo R, Campos B, García C. 2003. Evaluación endoscópica de hemorragia pulmonar inducida por el ejercicio en equinos de polo. Arch Med Vet, Valdivia 35(1): 109113.

8. Rojas C, Rodríguez C, Goic M, Mujica R. 2000. Prevalencia de hemorragia pulmonar inducida por el ejercicio post carrera en equinos fina sangre de carrera del club hípico de Santiago, mediante diagnóstico endoscópico y su relación con distintas variables. En: Resumen XI Congreso Nacional de Medicina Veterinaria. Santiago de Chile. p 912-913.

9. Sellnow L. 1997. Exercise-induced pulmonary hemorrhage. The Horse, USA 14(9): 20-26.

10. West JB, Mathieu-Costello O, Jones JH, Birks EK, Logemann RB, Pascoe JR, Tyler WS. 1993. Stress failure of pulmonary capillaries in racehorses with exerciseinduced pulmonary hemorrhage. J Appl Physiol 75: 1097-1109.

11. Zamalloa L. 1979. Contribución al estudio de la epistaxis en el Caballo Pura Sangre de Carrera en nuestro medio. Lima: Tesis de Bachiller. Facultad de Medicina Veterinaria, Univ. Nacional Mayor de San Marcos. 52 p. 